\title{
Réussir un développement urbain durable via la diversification
}

\author{
Paul-Émile David ${ }^{1}$ \\ Université de Moncton
}

\section{Introduction}

La pluralité est un concept de plus en plus présent au sein de notre société actuelle. Aux États-Unis, le concept traditionnel de «Melting Pot» se transforme continuellement en une image beaucoup moins homogénéisante, au point où nous faisons des efforts pour démasquer nos nombreux traits divergents. Cette pluralité est aussi apparente dans la structure industrielle de nos villes où la mono-industrialisation d'autrefois fait place à des régions urbaines ayant une plus grande variété d'entreprises se distinguant en âge, en vocation et en taille. En ayant une structure urbaine démontrant la diversification, l'inefficacité de la concentration industrielle peut être enrayée. Cette inefficacité est reflétée dans les propos de l'urbaniste Jane Jacobs ${ }^{2}$ qui mentionne : "An economy that contains few different sorts of niches for people's differing skills, interests and imaginations is not efficient. An economy that is unresourceful and unadaptable is not efficient. An economy that can fill few of the needs of its own people and producers is not efficient $\gg$.

Les villes ont un rôle important dans la production et l'accumulation de la richesse. Par contre, elles sont également des lieux où la désintégration des ressources naturelles, financières et humaines se fait voir. Pour les régions urbaines, un développement durable (c'est-à-dire un développement considérant les répercussions des décisions socio-économiques actuelles sur le bien-être des générations futures) pourrait assurer le respect des limites de l'environnement pour le court, le moyen et le long terme. Pour qu'un développement urbain durable se réalise, il est toutefois essentiel de pouvoir concevoir la ville comme un sys- tème vivant et ouvert. En ayant une approche systémique de la sorte, chaque action d'un agent socioéconomique pourrait être mieux évaluée sur une base globale, pour le présent ainsi que pour l'avenir.

L'objectif de ce texte est de souligner l'incapacité de la science économique traditionnelle à évaluer de façon efficace le rôle des villes au sein de notre société actuelle. Au lieu de poursuivre avec cette vision mécanique de la ville comme étant uniquement un lieu de transformation d'inputs en d'outputs, il est fondamental de la voir de façon organique, comme lieu de prédilection du développement global. C'est seulement lorsque cela se produira qu'il sera possible d'envisager un développement urbain qualifié de durable.

\section{Le raisonnement économique et le développement durable}

En science économique, les ressources naturelles, financières et humaines sont les facteurs servant à la production des biens et des services. Les ressources naturelles comprennent tout usage direct ou indirect des terres agricoles, des forêts, des étendues d'eau ainsi que de l'énergie éolienne, solaire et hydraulique. Les ressources financières (ou le capital) englobent les services procurés par la machinerie, les outils, les manufactures et tous les autres instruments permettant de produire d'autres biens, tout en haussant la productivité globale. Finalement, les ressources humaines réunissent l'ensemble des services rendus par des individus au plan manuel (physique) et non manuel (intellectuel).

Ces ressources sont utilisées comme inputs dans la fonction de production et ce, dans le but de les trans- 
former en outputs. La principale caractéristique des ressources est qu'elles sont relativement rares, nécessitant donc une allocation efficace afin d'en retirer les résultats souhaités.

La science économique est la discipline étudiant la façon dont nous pouvons satisfaire nos désirs illimités avec ces ressources qui sont qualifiées de rares. Nous devons gérer l'ensemble de nos ressources afin d'en retirer un niveau maximal de bien-être. C'est une question de choix : choisir les options nous rapportant le plus de satisfaction en respectant nos ressources limitées.

Une décision économique d'un agent sera efficace si l'accroissement de son bien-être (à la suite de cette décision) n'entraîne aucune baisse de bien-être d'un autre agent. Si une action entraîne une optimalité de bien-être, nous la considérons comme étant efficace du point de vue économique. En effet, ceci est tout à fait acceptable du point de vue économique, car les capacités des générations futures ne sont pas prises en considération. Toutefois, si nous adoptons une approche du développement dit "durable», nous prenons des décisions en tenant compte des effets que celles-ci auront sur l'ensemble de l'environnement, pour le présent ainsi que pour l'avenir.

Il est important de souligner que l'analyse économique n'est qu'une simulation dans un système fermé. Dans un système de la sorte, il est possible d'atteindre l'équilibre, c'est un résultat théorique. Par contre, dans un système ouvert (comme l'environnement urbain), nous ne cherchons qu'à retrouver la stabilité (l'équilibre ne pouvant être atteint). L'équilibre, et donc l'optimum de bien-être, sont impossibles à atteindre dans une situation semblable.

Promouvoir un développement durable, c'est viser l'harmonisation entre les agissements des êtres humains et l'environnement qui les entoure. C'est rechercher le juste niveau « entre ce que la technologie et la science nous permettent de faire et ce que l'on peut réellement faire ${ }^{3}{ }^{3}$; nous devons respecter la capacité de charge de l'environnement. Cela nous mène vers l'approche du développement urbain durable. Ceci se produit lorsque nous avons une vision systémique entre le développement durable, l'économie et le respect des ressources. Un tel développement dynamique et soutenable exige " une coopération étroite entre la population, les entreprises et les pouvoirs publics et des plans équitables, réalistes, conformes aux valeurs locales et aux attentes des intéressés. Citoyens, politiciens, urbanistes et corporations devraient être formés à travailler selon une telle approche ${ }^{4}$.

Selon Omar Aktouf ${ }^{5}$, la véritable question que nous devrions poser n'est pas de savoir où trouver des ressources indéfiniment renouvelables, mais plutôt quelles sont leurs utilisations. Ces utilisations deviennent plus efficaces lorsque la quantité limitée de ressources dans un environnement urbain est prise en considération. Comme le mentionne Dugas plus haut, la science et la technologie nous permettent d'effectuer plusieurs projets, mais il est important d'être soucieux de la capacité de charge de la planète et surtout, de ce qu'elle peut réellement accomplir.

\section{Promouvoir un développement durable, c'est viser l'harmonisation entre les agissements des êtres humains et l'environnement qui les entoure.}

\section{Le paradigme thermodynamique}

Dans cette optique, Aktouf nous offre le paradigme thermodynamique, permettant de mieux comprendre le destin qui nous est réservé si la tendance d'une surexploitation des mêmes ressources se maintient. Dans ce texte, nous présentons les arguments pour lesquels il est concevable que ce paradigme puisse décrire efficacement la mise en valeur des ressources au sein d'une région urbaine caractérisée par la diversité industrielle, par opposition à la concentration industrielle.

Nous posons l'hypothèse qu'une région urbaine est considérée comme un système vivant et ouvert. Afin de pouvoir survivre, ce système doit échanger de la matière et de l'énergie avec son environnement. Nous posons une deuxième hypothèse stipulant que cet échange se fait grâce aux lois de la thermodynamique; ce sont des lois de la physique basées sur les principes de la constance de la quantité d'énergie (ou de ressources) dans l'environnement et de l'entropie.

D'une façon plus simple, le premier principe exprime le fait que les ressources utilisables dans l'univers sont en quantité constante et non renouvelable à l'échelle 
humaine. Le deuxième principe stipule que l'évolution de ces ressources ne se fait qu'à sens unique, où les ressources utiles se transforment en ressources inutiles, c'est-à-dire que l'être humain peut faire presque tout, sauf « fabriquer » des ressources).

Le système vivant « naturel» transforme les ressources afin de se maintenir en vie; il y a l'entrée d'inputs et la sortie d'outputs. Pour tout système vivant, comme pour l'être humain, la quantité d'outputs sera toujours inférieure à celle des inputs. Si l'organisme humain effectuait le contraire de cela, son entropie serait accélérée et sa mort, hâtive. Pour tout système vivant, il est donc essentiel que la quantité d'outputs soit inférieure aux inputs, sinon nous serions en contradiction avec les lois de la thermodynamique.

Compte tenu du premier principe, l'ensemble de l'utilisation locale des ressources se traduit par la dégradation des ressources utilisables à l'échelle globale. Une décision au niveau local affecte donc le système au niveau global. Un système vivant « naturel» prélève régulièrement, jusqu'à son extinction irréversible, une certaine quantité de ressources vitales pour ralentir sa propre entropie (locale). En faisant cela, compte tenu du premier principe de la thermodynamique, l'entropie globale sera en hausse.

\section{En utilisant constamment les mêmes ressources et en ne modifiant pas ses activités industrielles, la structure mono-industrielle d'une ville ne peut lutter contre les effets cycliques de l'économie.}

\section{Comment freiner l'entropie dans une région urbaine?}

Selon Aktouf, pour ce qui est du monde des affaires, qui est un système artificiel, l'objectif est la maximisation des profits. Cela étant dit, les outputs d'une entreprise à but lucratif seront toujours supérieurs aux inputs. Ce scénario indique donc que l'entreprise capitaliste ne respecte pas les lois de la thermodynamique, comme tout autre système dans l'univers. La maximisation des profits entraîne des outputs supérieurs aux inputs (c'est-à-dire les ressources naturelles, financières et humaines) et, ainsi, selon les lois de la thermodynamique, cette transformation accélère l'entro- pie de l'entreprise (locale) et celle de son environnement au complet (globale).

Pour une entreprise dans une ville industriellement diversifiée, l'entropie peut être ralentie par le partage des ressources avec une entreprise d'une autre industrie. En puisant des ressources d'ailleurs (soit des nouveaux procédés, des idées...), l'entreprise peut ralentir son entropie de même que celle de son industrie. Dans une ville mono-industrielle, la dégradation de l'industrie en question est sans détour; Jacobs confirme ce constat lors de l'étude de nombreuses villes du monde entier. En utilisant constamment les mêmes ressources et en ne modifiant pas ses activités industrielles, la structure mono-industrielle d'une ville ne peut lutter contre les effets cycliques de l'économie. Nous pouvons tenter de freiner l'entropie urbaine, mais non de l'éliminer. Tel que mentionné plus haut, nous devons rechercher la stabilité, car l'équilibre est impossible dans un système ouvert.

Le fait d'avoir une diversité industrielle au sein d'une région urbaine procure plusieurs avantages aux entreprises, dont celui de pouvoir tirer profit des ressources en provenance des autres entreprises du même secteur d'activité ou d'un secteur différent. Le lien pouvant être établi entre la diversité et les lois de la thermodynamique semble être simple mais tout à fait véridique: plus une entreprise interagit avec différentes entreprises de diverses industries, plus elle aura la possibilité d'utiliser des ressources de divers endroits et de multiples façons. Si elle procède de cette façon, non seulement limitera-t-elle la surexploitation de ses propres ressources, mais elle réussira à freiner son entropie, car l'ensemble des ressources qu'elle utilise sera plus grand que son output.

\section{La coopération au sein d'un milieu urbain}

Une façon de ralentir l'épuisement trop rapide de nos ressources est donc possible via la coopération, qui est un élément essentiel au développement urbain durable. Selon la vision de Jacobs, les divers agents socioéconomiques au sein d'une ville diversifiée perçoivent qu'une situation de coopération est plus bénéfique pour leur bien-être individuel, de même que pour la ville en sa totalité, que la non-coopération. Les exemples présentés par Jacobs s'apparentent au concept de l'économie sociale. Dans une situation de chômage, d'exclusion sociale ou d'inaction du secteur public, les 
agents socio-économiques d'une communauté développent divers réseaux afin de satisfaire à leurs besoins : santé, transport public, culture, protection de l'environnement, coopératives de travailleurs et de consommateurs, etc. Il est possible qu'une mise en valeur des principes de ce «troisième secteur» permette le ralentissement de l'entropie urbaine et une meilleure utilisation des ressources. Une coopération étroite entre les divers agents socio-économiques est requise afin qu'un développement urbain durable soit envisageable.

La mise en valeur des diverses ressources dans une région urbaine peut donc être atteinte en se concentrant sur l'aspect coopératif de l'économie sociale. Plus particulièrement, une interaction entre diverses entreprises (de différentes industries) peut donner l'occasion d'avoir une meilleure gestion des ressources sans pour autant diminuer l'efficacité des entreprises. En ayant une structure urbaine axée sur la diversité industrielle, ce partage de ressources pourrait mieux s'effectuer.

\section{L'importance de la diversité au sein des régions urbaines}

Selon certains auteurs, la diversification industrielle est plus efficace pour une ville que la concentration industrielle, car lorsqu'une ville est dépendante d'un seul secteur, la prospérité de celui-ci sera jumelée à celle de la région métropolitaine en question. L'histoire économique nous fait part de plusieurs incidents où une ville a vu son statut économique s'amoindrir énormément avec le déclin de sa principale industrie. Aux États-Unis, la forte concentration de l'industrie de l'acier au sein de plusieurs petites villes s'est présentée comme un élément explicatif du déclin de celles-ci lorsque les Américains ont eu à faire face à une accentuation de la concurrence étrangère et à des politiques commerciales défavorables à leurs exportations.

Si une ville ne dépend que de certaines ressources naturelles (la forêt, les mines de charbon, etc.), sa prospérité sera jumelée à celle des ressources en question. La même affirmation peut être exprimée des villes uniquement axées sur des ressources humaines ou financières. Une ville industriellement diversifiée implique une utilisation plus équitable des nombreuses ressources, par opposition à une ville mono- industrielle épuisant continuellement les mêmes ressources et utilisant les mêmes procédés.

Avec la diversification industrielle, il est envisageable que l'entropie urbaine soit freinée et qu'il y ait croissance. En effet, un environnement urbain ayant plusieurs entreprises produisant des biens différenciés est plus apte à bénéficier d'une croissance économique qu'un autre où il n'y a qu'une concentration industrielle. Cela se produit du fait que, dans une région urbaine ayant plusieurs petites entreprises, plus d'activités entrepreneuriales et une infrastructure bien établie, la compétitivité sera plus grande ainsi que la coopération au niveau des ressources.

\section{Les approches mécanistes et organiques}

Selon certains chercheurs, le dynamisme des villes dépend en grande partie du degré de renouvellement, de modernisation et d'adaptation de leurs bases économiques industrielles. Cela oppose donc le concept de la concentration à celui de la diversification. À partir des théories du management, les approches «mécanistes » et « organiques » nous permettent d'expliciter davantage cette dichotomie existant entre la mono-industrialisation et la diversification industrielle.

L'approche mécaniste pose pour hypothèse que nous vivons dans un environnement pouvant être qualifié de stable, routinier et connu, bref un système fermé. Par contre, si de fortes fluctuations se produisent au sein de l'économie, l'entreprise ayant une approche mécaniste peut se retrouver dans une situation difficile en raison de sa structure rigide et sensible aux fluctuations. La même chose peut être dite d'une ville mono-industrielle utilisant ses ressources de façon peu variée.

\section{Avec la diversification industrielle, il est envisageable que l'entropie urbaine soit freinée et qu'il y ait croissance.}

Par opposition, l'entreprise ayant une approche plutôt organique est plus souple que celle qui est mécaniste. L'organisation est ouverte et recueille des ressources d'endroits et de lieux divergents afin de s'adapter à la conjoncture socio-économique. Cela s'apparente à une région urbaine où il y a diversification industrielle. Le partage et la coopération entre divers agents font en 
sorte qu'il y a peu de stagnation. Les ressources sont variées et utilisées de différentes façons. L'approche mécaniste tend à manquer d'efficacité, sauf lorsque les tâches et l'environnement sont à la fois considérés simples et prévisibles (c'est-à-dire dans un système fermé).

\section{Que ce soit la science, la culture, l'économie ou l'innovation, elles trouvent toutes un lieu d'expression privilégié dans la ville ayant un degré élevé de diversification.}

Les sociétés évoluent de façon organique; aux rapports simples caractérisant les sociétés élémentaires succèdent les rapports plus complexes des sociétés modernes. Dans les rapports simples, les fonctions sociales sont peu diversifiées. Nous parlons alors de structures segmentaires et de solidarité mécanique. Par opposition, les sociétés plus complexes se distinguent moins de l'homogénéité organique du groupe que de la complémentarité fonctionnelle de ses membres. Nous avons donc une solidarité organique nous permettant d'encourager la diversité des idées.

Les villes mono-industrielles encouragent l'exode des gens plutôt que le contraire. L'intégration sociale au sein de celles-ci est limitée par un choix restreint d'emplois. L'exemple de certaines communautés pêcheuses du Canada atlantique, où les jeunes gens décident de quitter pour de meilleures possibilités d'emplois, est emblématique de ce syndrome. De toute évidence, refaire le travail de ses parents n'est pas perçu comme un choix mais plutôt comme une obligation pour les jeunes demeurant dans ces communautés fortement dépendantes de la pêche. Que ce soit la science, la culture, l'économie ou l'innovation, elles trouvent toutes un lieu d'expression privilégié dans la ville ayant un degré élevé de diversification.

Afin d'avoir un développement urbain véritablement durable, il est essentiel de maintenir un équilibre entre trois pôles : l'efficacité économique, l'intégrité écologique et l'équité sociale. Par contre, un ajout doit être effectué au niveau de cette approche : nous devons inclure la dimension éthique. En effet, il est primordial de prendre en considération les relations entre les générations actuelles et futures, les régions riches et pauvres de même qu'entre les diverses classes socia- les ${ }^{6}$. La mise en valeur de nos nombreuses ressources (naturelles, financières et humaines) doit donc passer par un raisonnement éthique; ce raisonnement est possiblement le facteur clé pour transformer un raisonnement «purement économique» en un raisonnement « durable ».

\section{Conclusion}

Notre conclusion débute avec une citation pouvant résumer en quelque sorte l'importance que représente la diversité pour tout système vivant (incluant les régions urbaines) : "La loi de la variété requise est reliée aux concepts de différenciation et d'intégration. Cette loi stipule que les mécanismes régulateurs internes d'un système doivent être aussi variés que l'environnement avec lequel il tente de composer, car ce n'est qu'en intégrant la variété requise aux contrôles internes qu'un système peut faire face à la variété et aux défis de son environnement. Un système qui s'isole de la variété du milieu dans lequel il se trouve tend à s'atrophier, perd de sa complexité et voit disparaître ses traits distinctifs. C'est ainsi que la variété requise constitue une caractéristique importante de tous les systèmes vivants $»^{7}$.

Cette notion de la diversité requise pour les systèmes vivants s'apparente, au dire de Jacobs, à la nécessité d'avoir de la diversité industrielle au sein d'un environnement urbain. La diversité (sous toutes ses formes) est exigée pour les systèmes vivants de même que pour les régions métropolitaines où l'hétérogénéité doit non seulement se retrouver au sein de la structure industrielle, mais aussi au niveau des nombreuses ressources (naturelles, financières et humaines) utilisées. Il est important, par contre, de constater que cette diversité doit être fondée sur un raisonnement éthique de notre société et de ses capacités (présentes et futures). En prônant un développement urbain durable, il est possible d'intégrer cette éthique en ce qui concerne nos décisions traitant de l'urbanisation, de la diversité et de l'utilisation que nous faisons de nos nombreuses ressources.

Un développement urbain durable se fonde sur des principes permettant un mode de vie respectant les capacités de l'environnement. En recherchant l'amélioration de la qualité de la vie, le respect des limites de la capacité de charge de la planète, une plus grande coopération entre les divers agents socio-économiques 
ainsi que la préservation de la diversité de la terre, il est envisageable qu'un développement urbain durable, dynamique et soutenable se produise et qu'une utilisation plus saine de nos ressources se réalise.

La présence de concentration industrielle peut se comparer au non-respect d'une vision durable. Le manque de coopération et de partage des ressources entre les divers agents socio-économiques au sein d'une ville mono-industrielle peut résulter en une situation de déclin. Il est envisageable que l'absence de diversité industrielle soit une raison possible pour le manque de dynamisme au sein de plusieurs villes.

La notion du développement urbain durable invite donc les agents socio-économiques à se réinventer face au raisonnement néo-libéral si dominant dans la conjoncture actuelle. En considérant les impératifs de l'écologie, de l'économie, du social et surtout de l'éthique dans leurs nombreuses décisions, il est espéré que ces agents réussissent ainsi à valoriser les ressources (naturelles, financières et humaines) au sein d'une région urbaine industriellement diversifiée, pour le présent ainsi que pour l'avenir.

\section{Notes et références}

1 Paul-Émile David est professeur adjoint au département d'économie de l'Université de Moncton.

2 Jacobs, J. (1984), Cities and the Wealth of Nations, New York, Vintage, p. 17.

3 Dugas, P. (1994). « Le développement durable ou comment atteindre l'harmonie ", Forêt Verte, Planète Bleue, p. 154.

4 UICN/PNUE/WWF (1991), Sauver la planète: stratégie pour l'avenir de la vie, Suisse, Gland, p. 116.

5 Aktouf, O. (1995), « Rationalité économique néo-classique et entropie : une limite thermodynamique à la "Libre Entreprise ?" ", Cahier de Recherche du CETAI-HEC Montréal, NRJ-CR-95-01 (octobre).

6 Vaillancourt, J.-G. (1995), « Penser et concrétiser le développement durable », Ecodécision (hiver), p. 24-29.

7 Morgan, G. (1989), Images de l'organisation, Québec, Les Presses de l'Université Laval, p. 43. 